\title{
sensors
}

ISSN 1424-8220

(C) 2007 by MDPI

www.mdpi.org/sensors

Full Research Paper

\section{Carbon-based Composite Electrodes: Preparation, Characterization and Application in Electroanalysis}

\author{
Ioana Corb ${ }^{1}$, Florica Manea ${ }^{1, *}$, Ciprian Radovan ${ }^{2}$, Aniela Pop ${ }^{1}$, Georgeta Burtica ${ }^{1}$, \\ Plamen Malchev ${ }^{3}$, Stephen Picken ${ }^{3}$ and Joop Schoonman ${ }^{3}$ \\ 1 "Politehnica" University of Timisoara, P-ta Victoriei, nr.2, 300006, Timisoara, Romania. \\ E-mails: ioana.corb@chim.upt.ro, florica.manea@chim.upt.ro, aniela.pop@chim.upt.ro, \\ georgeta.burtica@chim.upt.ro
}

2 West University of Timisoara, Str. Pestalozzi, Nr.16, 300115, Romania. E-mail: radovan@cbg.uvt.ro 3 Delft University of Technology, 2600 GA Delft, The Netherlands.

E-mails: p.g.malchev@tnw.tudelft.nl,s.j.picken@tnw.tudelft.nl,j.schoonman@tnw.tudelft.nl

* Author to whom correspondence should be addressed.

Received: 2 October 2007 / Accepted: 5 November 2007 / Published: 5 November 2007

\begin{abstract}
Electrodes based on carbon, i.e., expanded graphite (20\%, wt.)-epoxy composite (20EG-Epoxy) and expanded graphite (20\%, wt.)-polystyrene composite (20EG-PS) have been prepared, characterized using scanning electron microscopy (SEM) and cyclic voltammetry $(\mathrm{CV})$, and tested as anodic sensors. The electrodes exhibited good mechanical resistance and low electrical resistances. Scan rate dependent cyclic voltammetry responses at 20EG-Epoxy and 20EG-PS composite electrodes, which were exemplified for thiourea (TU), a toxic sulphur organic compound selected as testing target analyte in $0.1 \mathrm{M} \mathrm{Na}_{2} \mathrm{SO}_{4}$ supporting electrolyte, were investigated. The obtained voltammetric data were in accordance with those for a random array of microelectrodes. The voltammetric and chronoamperometric detection results of TU in tap water samples, without a supplementary addition of supporting electrolyte, at 20EG-Epoxy electrode proved its use for direct analysis of environmental samples.
\end{abstract}

Keywords: carbon-based composite electrode, microelectrode array behavior, thiourea, electroanalysis 


\section{Introduction}

Carbon has presented the grown interest as electrode material because it exhibits many advantages, including good electrical conductivity, chemical inertness and wide potential range [1, 2]. Carbonbased materials such as graphite, carbon fibers, etc. have largely been used in the last time as the conductive phase in composite materials suitable for electrochemical sensors [3-5]. The use of composites based on conductive phase dispersed within insulating polymer matrices as electrodes has led to important advances in developing sensor devices [6-13]. The composite materials consisted of the combination of two or more materials, in which each individual component exhibit its original nature should be seen as a whole with the distinctive chemical, mechanical and physical peculiarities [6]. Electrodes obtained by using a mixture of particulate conductive carbon phase and an insulating matrix represent an attractive approach to fabrication electrochemical sensors, whose surface can be renewed by polishing $[3,7]$. The insulating phase has shown a grown interest for investigation in order to decrease the electrical resistance and to improve the electrochemical behavior of the composite.

In this work certain new carbon-based composite electrodes, i.e., expanded graphite (20\%, wt.)epoxy (20EG-Epoxy), expanded graphite (20\%,wt.)-polystyrene (20EG-PS), were elaborated and evaluated for the electroanalytical utility. Scaning electron microscopy (SEM) has been used to determine the physical distribution of the carbon particles within insulating matrices (epoxy or polystyrene). The electrochemical characterization of these composite electrodes were carried out by using cyclic voltammetry (CV) and chronoamperometry (CA). Thiourea (TU) was chosen as target pollutant for electrochemical assessment of the behaviour and the use of the studied carbon-based composite electrodes as available sensors and their application in electroanalysis. Thiourea is only an example of a presumptive toxic compound regarded as representative sulphur-containing pollutant in water [14,15 ]. It was selected as a very adequate analyte for put in evidence the microelectrode array behaviour of the elaborated sensing device, at the same time the principal aim of the work.

\section{Results and Discussion}

\subsection{Surface characterization}

SEM images of fractured surfaces of both composites were taken to qualify the bulk distribution and the structure of the conductive carbon filler (Figure 1a, b).

The appearance of the fractured surfaces of both composites containing is very similarly. Generally, the graphite flakes are well distributed within both organic matrices. However, some differences that can be explained by the different preparation conditions are present. A layering of the graphite flakes parallel to the surfaces of the plate is visible for the 20EG-Epoxy electrode, due to the pressing of the plate in the hot press (Figure 1a). Such layering is absent in the 20EG-PS electrode that has been prepared by casting a film from a solution. For this composite one can distinguish closely spaced zones of graphite flakes with a random orientation (Figure $1 \mathrm{~b}$ ). This observation is also confirmed by the different resistivities measured for the two composites. A random orientation of the conductive filler, as in the case of the 20EG-PS composite, provides with better conditions for conduction of the electrical current across the thickness of the electrode in contrast to the case when a layering parallel to the surfaces of the electrode exists, as for the 20EG-Epoxy composite. This leads to a slightly lower 
resistance of the 20EG-PS composite $(5.8 \Omega)$ than the one of the 20EG-Epoxy (7.3 $\Omega$ ). In conclusion the explored materials available in elaboration of the investigated electrodes showed good mechanical strength with relatively low electrical resistivity. The different detection results in their application for electrochemical sensing devices can be entirely related to the nature of the matrix.

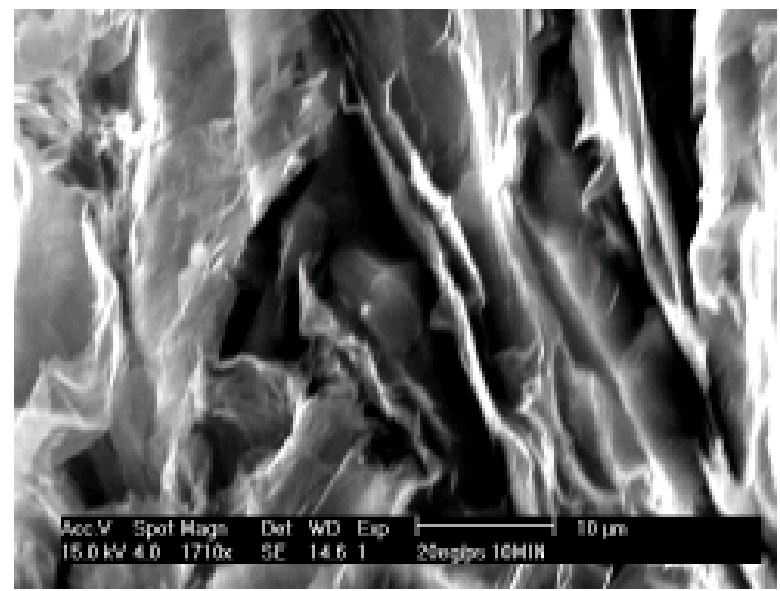

a)

Figure1. SEM image of a fractured surface of: a) 20EG-Epoxy;

b) 20EG-PS; black zone represent expanded graphite.

\subsection{Electrochemical behavior}

Several literature data underline that the carbon based composite electrode can be regarded either ordered (array) or randomized microelectrode ensembles [2, 7, 16]. Under these circumstances, the diffusion flux toward microelectrodes ensembles depend strongly on the experimental timescale. Figure 2 shows the cyclic voltammetric responses at each studied carbon-based composite electrode in $0.1 \mathrm{M} \mathrm{Na}_{2} \mathrm{SO}_{4}$ supporting electrolyte containing $1 \mathrm{mM} \mathrm{TU}$, used as testing analyte.

The low signal-to-background ratio was obtained and all studied carbon-based composite electrodes provided the irreversibility of TU electrochemical oxidation.

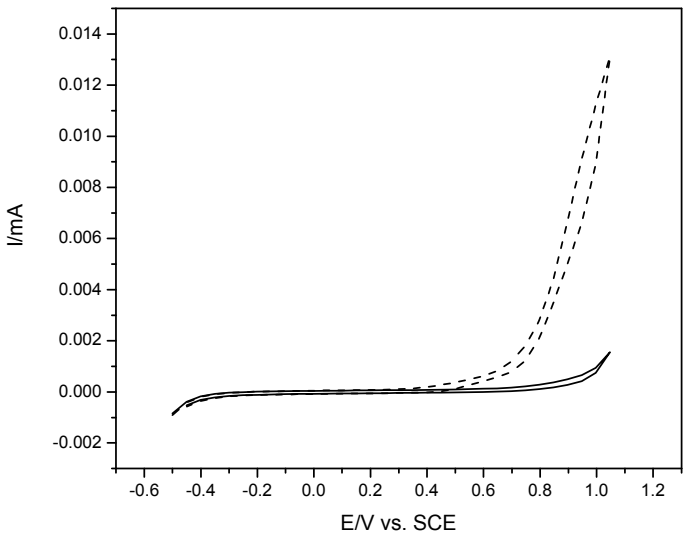

a)

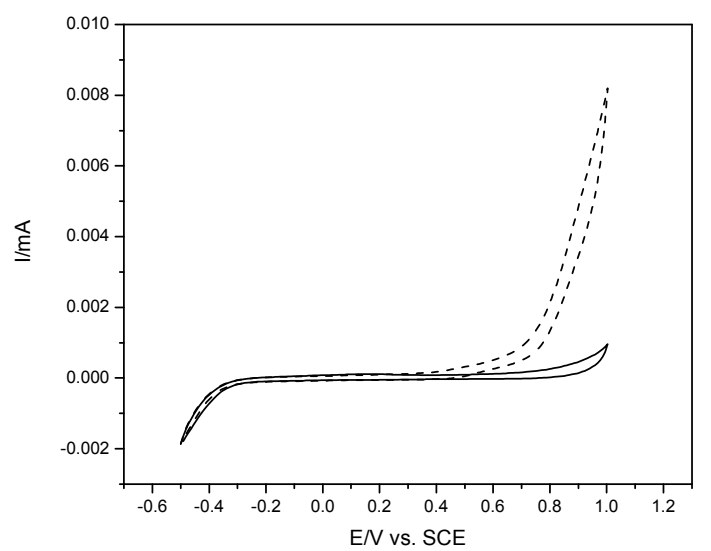

b)

Figure 2. Cyclic voltammograms for $1 \mathrm{mM}$ thiourea in $0.1 \mathrm{M} \mathrm{Na}_{2} \mathrm{SO}_{4}$ supporting electrolyte (dotted line) and $0.1 \mathrm{M} \mathrm{Na}_{2} \mathrm{SO}_{4}$ supporting electrolyte (solid line) at different carbon-based composite electrodes: a) 20EG-Epoxy, b) 20EG-PS, scan rate was $0.05 \mathrm{~V} \cdot \mathrm{s}^{-1}$. 
The specific micro-dispersed nature of the investigated carbon-based composite electrode suggested a presumptive way to discuss the corresponding electrochemical data considering the typical aspects regarding the behaviour of randomized or array structured microelectrodes systems.

For some carbon-based composite electrode, it has been proved the heterogeneous electroactivity with distinct microzones of a significantly higher electrochemical activity [7]. The voltammetric responses of carbon-based composite electrodes are sometimes similarly to that found for ,edge effect", which contribute significantly to the Faradaic current. However, these structured materials, as in our investigated composites, are not rigorously arrays but are truly randomised ensembles, with the size, shape and inter-microelectrode separation distributed over a wide range. The random ensembles of microelectrodes can have array behaviour in certain situation [7], an important characteristic expected to manifest similarly for our explored electrodes. In the preparation of carbon-based composites with microelectrode array behaviour, certain important requirements should be met. The main requirement is that the gaps between individual conductive carbon microzones should be much larger than its radius, when spherical diffusion dominates mass transport. The closely spaced carbon microzones array will behave similar to a macroelectrode (linear diffusion controlled mass transport) because of the diffusion layer overlap [16-24]. Another requirement is subjected to the insulating matrix to prevent current leakage, which resulted in the distortion of the cyclic voltammetry [6]. Microelectrode arrays exhibit the advantages of single microelectrodes, e.g., reduced ohmic drop and charging current, the lower detection limit and better sensitivity. In the same time, their use overcome the disadvantages of single microelectrode subjected to low current outlet and the high susceptibility to the electrochemical noise [19].

Because the possibility to observe microelectrode array behavioural aspects depends the size, geometry and the gaps between microelectrodes, but also on the response time of the electrode, the scan rate in the cyclic voltammetry was systematically varied over a wide range of scan rates. Such, scan rate effect on the voltammograms can be use as a first diagnostic criterion of the electrochemical behavioural characteristics.

Figure 3 illustrates the effects of the scan rate on the cyclic voltammograms of each investigated our composite. The scan rate is systematically varied from 0.001 to $0.05 \mathrm{~V} \cdot \mathrm{s}^{-1}$ in $1 \mathrm{mM}$ TU containing $0.1 \mathrm{M} \mathrm{Na}_{2} \mathrm{SO}_{4}$ supporting electrolyte. For both composites electrodes, the scan rate effect is relatively slight. In addition, for these types of composites a current plateau has been observed when the scan rate was equal or lower than approximately $0.05 \mathrm{~V} \cdot \mathrm{s}^{-1}$. This result, regarding a limiting current suggests that for the slower scan rates, a pseudo-steady state response has been achieved with little hysteresis effect between the forward and backward branches of the cyclic voltammograms. This behavior is in accordance with a microelectrode array behaviour of the investigated composite electrodes.

\subsection{Electrochemical detection in solution of low ionic strength}

The ability of the microelectrode array to deliver the current responses in the absence of any supporting electrolyte is an attractive feature when they are compared with the conventional macroelectrodes [21]. There are several reason subjected to analytical studies on nondilute samples in the absence of any supporting electrolyte, i.e., the direct analysis in low ionic strength solutions that 
make analysis more facile, eliminating the possible interference of the supporting electrolyte and the possibility of extending the upper concentration limit of the analyte under investigation.

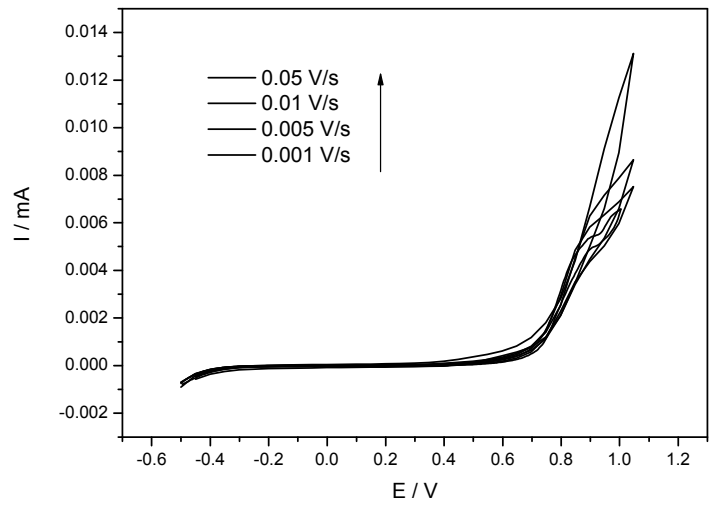

a)

Figure 3. Cyclic voltammograms for $1 \mathrm{mM}$ TU solution at: a) 20EG-Epoxy composite electrode; b) 20EG-PS composite electrode, supporting electrolyte was $0.1 \mathrm{M} \mathrm{Na}_{2} \mathrm{SO}_{4}$.

Based on the first preliminary evidence of the electrochemical behavior of the studied carbon-based composite electrode, it was found that 20EG-Epoxy electrode, selected as individual example in the sensing application, represented an optimal working electrode for analytical purposes, e.g., TU detection in tap water, without supporting electrolyte, a second certification of the microelectrode array behaviour. The responses obtained at 20EG-Epoxy in the absence of supporting electrolyte and for different concentrations of TU in tap water are presented in Figure 4. This depicts the voltammetric responses obtained at the electrode at scan rate of $0.05 \mathrm{~V} \cdot \mathrm{s}^{-1}$, which was chosen to keep the negligible ohmic drop. The anodic current recorded at $0.9 \mathrm{~V}$ vs. SCE increases with increasing of TU in tap water and a relative low hysteresis was observed. Inset of Figure 4 shows that the anodic current from forward branches of CVs recorded at $0.9 \mathrm{~V} / \mathrm{SCE}$ depends linearly on the TU concentration in the range 0.1-1.0 mM, with a correlation coefficient better than 0.99 and the sensitivity of $0.01426 \mathrm{~mA} \cdot \mathrm{mM}^{-1}$.

The anodic detection of thiourea is not specifically, other electroactive species offer amperometric signals in the same potential range, but remains an very adequate reference example for anodic detection in the circumstances of an array behaviour of the used carbon-based composite electrode.

A typical hydrodynamic chronoamperometry (Figure 5) was obtained by successively adding TU to a quiescent tap water. The applied potential was set at $+0.9 \mathrm{~V}$ vs. SCE and the response time was about 50 seconds. The inset of Figure 5 shows the calibration plot of successive additions of $0.5 \mathrm{mM}$ TU to tap water. The calibration plot of current versus TU concentration was linear over the concentration range of 0.5 to $4 \mathrm{mM}$, with the correlation coefficient of 0.98 . The sensitivity of this cronoamperometric assessment of $0.00348 \mathrm{~mA} \cdot \mathrm{mM}^{-1}$ is lower compared to $\mathrm{CV}$ analytical data, probably because of some cumulative and remanent adsorption effects on the surface of the electrode. 


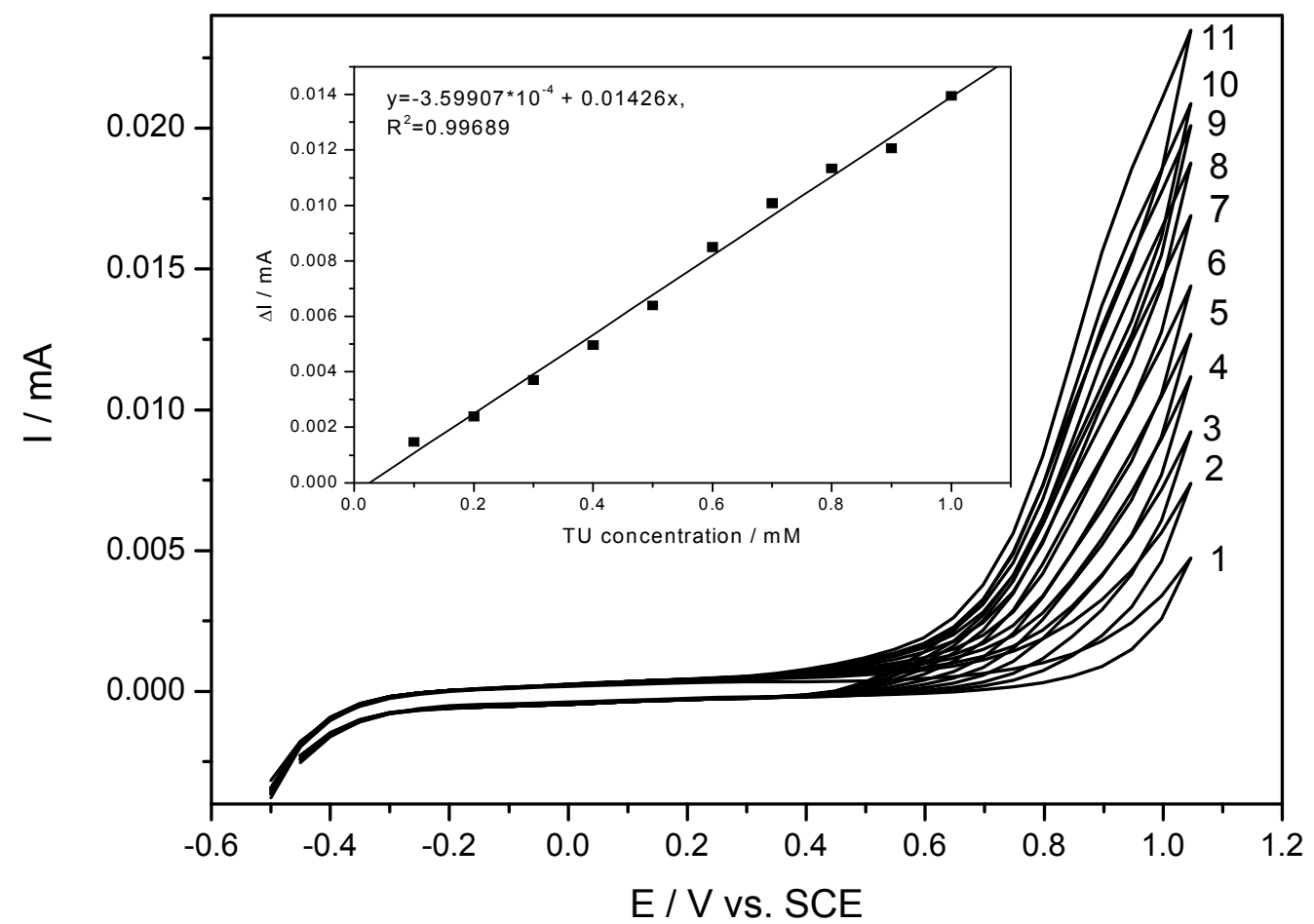

Figure 4. Cyclic voltammograms of 20EG-Epoxy in the tap water and the presence of different TU concentrations:1-0 mM; 2-0.1 mM; 3-0.2 mM; 4-0.3 mM; 5-0.4 mM; 6-0.5 mM; 7-0.6 mM; 8-0.7 mM; 9-0.8 mM; 10-0.9 mM; 11-1 mM; potential scan rate $0.05 \mathrm{Vs}^{-1}$;. Inset: the calibration plot of anodic current from forward branches of $\mathrm{CV}$ s recorded at $0.9 \mathrm{~V} / \mathrm{SCE}$ vs. TU concentrations.

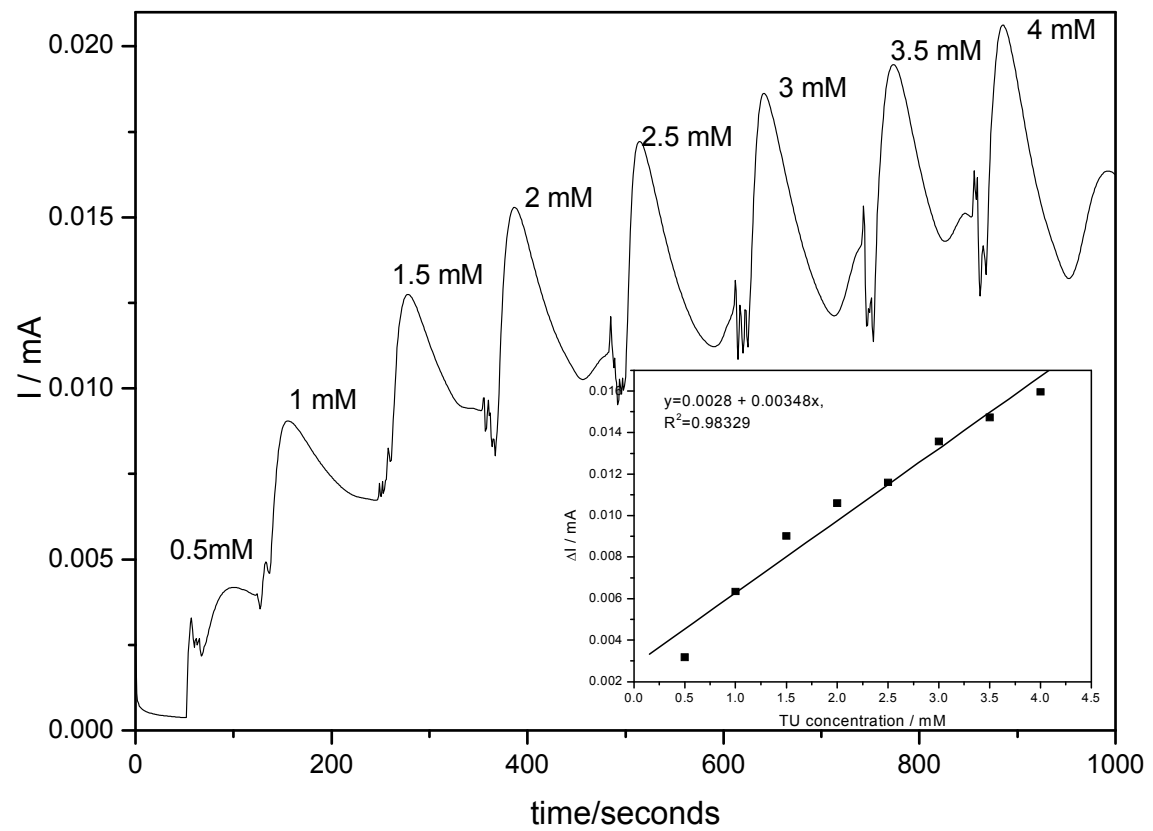

Figure 5. Chronoamperometric records obtained at 20EG-Epoxy for successive additions of $0.5 \mathrm{mM}$ TU in tap water, without supporting electrolyte; working potential:+0.9 V/SCE; Inset: the calibration plot of current recorded after 50 seconds vs. TU concentration. 
At the potential value of $0.9 \mathrm{~V}$ vs. SCE, a linear relationship between the amperometric signal and TU concentration was obtained in the range 0.1 to $1 \mathrm{mM}$ using cyclic voltammetry and between 0.5 and $4 \mathrm{mM}$ using chronoamperometry (CA) and successive addition of target compounds in hydrodynamic conditions. Under these experimental conditions, no cleaning or pretreatment of the electrode after each addition of TU was required. The analytical parameters are presented in Table 1. The lowest limit of detection (LOD) was evaluated based on 3 sigma method (calculated as 3 times the standard deviation of the blank over the sensitivity) [9]. The reproducibility of the data at the EG-PS electrode using the above-mentioned techniques was evaluated for three replicates measurements of TU detection. The relative standard deviations (RSD) gathered in Table 2 show the good reproducibility of the electrode.

Table 1. The analytical parameters of amperometric detection of TU at an EG-PS electrode using different techniques.

\begin{tabular}{cccccc}
\hline $\begin{array}{c}\text { Technique } \\
\text { used }\end{array}$ & $\begin{array}{c}\text { Concentration } \\
\text { range }(\mathrm{mM})\end{array}$ & $\begin{array}{c}\text { Electrode sensitivity } \\
\left(\mathrm{mA} \cdot \mathrm{mM}^{-1}\right)\end{array}$ & $\begin{array}{c}\text { Correlation } \\
\text { coefficient } \\
\left(\mathrm{R}^{2}\right)\end{array}$ & $\begin{array}{c}\text { LOD } \\
(\mathrm{mM})\end{array}$ & $\begin{array}{c}\text { RSD } \\
(\%)\end{array}$ \\
\hline $\mathrm{CV}$ & $0.1-1$ & 0.01426 & 0.9992 & 0.02 & 2.5 \\
$\mathrm{CA}$ & $0.5-4$ & 0.00348 & 0.9832 & 0.2 & 3.2 \\
\hline
\end{tabular}

\section{Experimental Section}

The composite electrodes were prepared from low viscosity polystyrene pellets (PS N2000, Crystal from Shell) or two-component epoxy resin (LY5052, Araldite) mixed with conductive expanded graphite (EG) fillers powder (Conductograph, SGL Carbon). To prepare 20EG-PS, the conductive expanded graphite filler (EG) was added to a toluene (Fluka) solution of PS N2000 with constant stirring. Ultrasound was used for about $2 \mathrm{~h}$ to mix well the components. The obtained viscous mixture was cast into a film using a Teflon mold and the toluene was let to evaporate first at room temperature for $48 \mathrm{~h}$ and later in a vacuum oven at $50^{\circ} \mathrm{C}$ for $24 \mathrm{~h}$. The final thickness of the obtained film was about $1 \mathrm{~mm}$. In the case of the 20EG-Epoxy composite it was not possible to add the full amount of EG to the matrix resin directly, due to the high surface area of the graphite flakes, therefore, the mixing was performed in a roll-mill at room temperature. The two parts of the epoxy were mixed together and the full amount of the EG was added in steps forming a thick paste. Then the epoxy was cured in a hot press at $80^{\circ} \mathrm{C}$ for 40 minutes. Simultaneously the material was shaped in a plate of 1 $\mathrm{mm}$ thickness. The plate was slowly cooled down (for about $12 \mathrm{~h}$ ) to the room temperature without removing the applied pressure. The prepared carbon-based composites showed good mechanical strength and low electrical resistance (Table 2). 
Table 2. The electrical resistance of the material of each carbon-based composite electrode

\begin{tabular}{cc}
\hline Carbon -based composite type & $\begin{array}{c}\text { Electrical resistance* } \\
(\Omega)\end{array}$ \\
\hline 20EG-Epoxy & 7.3 \\
20EG-PS & 5.8 \\
\end{tabular}

*evaluated for the thickness of $1 \mathrm{~mm}$ and over an area of $81 \mathrm{~mm}^{2}$

Plates with a surface area of $9 \mathrm{~mm}^{2}$ were cut from all compositions put on a glass supports and electrical contacts were made using a silver paint. The electrodes were isolated on the sides by epoxy resin. Prior to use, this working electrode was gradually cleaned, first polished with abrasive paper and then on a felt-polishing pad by using $0.3 \mu \mathrm{m}$ alumina powder (Metrohm, Switzerland) in distilled water for 5 minutes and rinsing with distilled water.

A Scanning Electron Microscope (Philips CM30T) was used to observe the working electrode surface.

The electrochemical performances of these electrodes were studied by cyclic voltammetry $(\mathrm{CV})$ and chronoamperometry (CA). Subsequently, an electrochemical pretreatment by three repetitive cyclings between $-0.5 \mathrm{~V}$ to $1.25 \mathrm{~V}$ vs. $\mathrm{SCE}$ in $0.1 \mathrm{M} \mathrm{Na}_{2} \mathrm{SO}_{4}$ supporting electrolyte was performed. All measurements were carried out using an Autolab potentiostat/galvanostat PGSTAT 302 (Eco Chemie, The Netherlands) controlled with GPES 4.9 software and a three-electrode cell, with a saturated calomel electrode as reference electrode, a platinum counter electrode and a carbon-based composite as working electrode.

Thiourea and sodium sulphate were analytical grade from Merck, and the solutions were freshly prepared with double-distilled water.

\section{Conclusions}

This work demonstrated that carbon-based composites electrodes using epoxy and polystyrene matrix showed good mechanical resistance and slight different low electrical resistances depended on the insulating matrix and preparation method. From the perspective of electroanalytical applications, 20EG-Epoxy and 20EG-PS exhibited microelectrode array behaviour. It was possible to observe practically the low hysteresis of the cyclic voltammograms and an equivalence of steady state responses in quiescent solution at the scan rates of the several orders faster than for conventional macroelectrode.

20EG-Epoxy composite electrode gave amperometric responses for analytical use, and in some adequate circumstances no need a supplementary supporting electrolyte, as example a very dilute electrolytic composition of tap water being completely satisfactory. For the determination of TU in tap water by using $\mathrm{CV}$ and $\mathrm{CA}$ procedures, linear plots of current vs. concentration were obtained in the concentration range of TU between 0.1 to $1 \mathrm{mM}$, and 0.5 to $4 \mathrm{mM}$ respectively, with LOD of $0.02 \mathrm{mM}$ and $0.2 \mathrm{mM}$, without the need of a renewed surface between successive runs. This electrode proffered 
the possibility of analysis directly in environmental samples, e.g., tap water with $\mathrm{TU}$, or more generally with an organic load.

The anodic detection of thiourea remains an adequate example for anodic detection in the circumstances of array behaviour of the used carbon-based composite electrode.

\section{Acknowledgements}

Funding for this project was provided by the Romanian Excellence Research for Young Researcher CEEX-ET Program: Smart sensors for waste water quality monitoring, Grant CNCSIS Type A Code No. 1377 and Romanian National Research of Excellence Program- CEEX, Grant 62/03.10.2005 Advanced Treatment Technologies for Recycling Industrial Wastewater/RIWATECH.

\section{References and Notes}

1. Tu, Y.; Lin, Y.; Yantasee, W.; Ren, Z. Carbon Nanotubes Based Nanoelectrode Arrays: Fabrication, Evaluattion, and Application in Voltammetric Analysis. Electroanalysis 2005, 17, 79-84.

2. Wang, J. Analytical Electrochemistry; VCH Publishers: New York, NY, 2000; Chapter 4, pp. 100-139.

3. Rassaei, L.; Sillanpaa, M.; Bonne, M. J.; Marken, F. Carbon Nanofiber-Polystyrene Composite Eectrodes for Electroanalytical Processes. Electroanalysis 2007, 19(14), 1461-1466.

4. Zhu, L.; Tian, C. Zhai, J; Yang, R. Sol-gel Derived Carbon Nanotubes Ceramic Composite Electrodes for Electrochemical Sensing. Sens. Actuators B. 2007, 125, 254-261.

5. Pumera, M.; Merkoci, A.; Alegret, S. Carbon Nanotube-epoxy Composites for Electrochemical Sensing. Sens. Actuators B. 2006, 113, 617-622.

6. Ballarin, B.; Cordero-Rando, M. M.; Blanco, E.; Hidalgo-Hidalgo De Cisneros, J. L.; Seeber, R.; Tonelli, D. New Rigid Conducting Composites for Electrochemical Sensors. Collect. Czech. Chem. Commun. 2003, 68, 1420-1436.

7. Ramirez-Garcia, S.; Alegret, S.; Cespedes, F.; Forster, R. J. Carbon Composite Electrodes: Surface and Electrochemical Properties. Analyst 2002, 127, 1512-1519.

8. Gabay, T.; Ben-David, M.; Kalifa, I.; Sorkin, R.; Abrahams, Z. R.; Ben-Jacob, E.; Hanein, Y. Electro-chemical and Biological Properties of Carbon Nanotubes based Multi-electrode Arrays. Nanotechnology 2007, 18, 035201:1-035201:6.

9. Manso, J.; Mena, M.L.; Yanez-Sedeno, P; Pingarron, J. Electrochemical Biosensors Based on Colloidal Gold-nanotube Composite Electrodes. J. Electroanal. Chem. 2007, 603 (1), 1-7.

10. Sun, D.; Zhu, L.; Zhu, G. Glassy Carbon Ceramic Composite Electrodes. Anal. Chim. Acta 2006, 564, 243-247.

11. Mendes, R. K.; Cervini, P.; Cavalheiro, E. T. G. The Use of a Graphite-Castor Oil Polyurethane Composite Electrode for the Determination of Hidroquinone in Photographic Developers. Talanta. 2006, 68, 708-712.

12. O'Hare, D.; Macpherson, J. V.; Wilson, A. On the Microelectrode Behaviour of GraphiteEpoxy Composite Electrodes. Electrochem. Commun. 2002, 4, 245-250. 
13. Marken, F.; Gerrard, M. L.; Mellor, I. M.; Mortimer, R. J.; Madden, C. E.; Fletcher, S.; Holt, K.; Foord, J. S.; Dahm, F. Voltammetry at Carbon Nanofiber Electrodes. Electrochem. Commun. 2001, 3, 177-180.

14. Manea, F.; Radovan, C.; Schoonman, J. Amperometric Determination of Thiourea in Alkaline Media on a Copper Oxides-Copper Electrode. J. Appl. Electrochem. 2006, 36, 1075-1081.

15. Spataru, N.; Spataru, T.; Fujishima, A. Voltammetric Determination of Thiourea at Conductive Diamond. Electroanalysis. 2005, 17(9), 800-805.

16. Stulik, K.; Amatore, C.; Holub, K.; Marecek, V.; Kutner, W. Microelectrodes. Definitions, Characterization, and Applications. Pure Appl. Chem. 2000, 72(8), 1483-1492.

17. Simm, A. O.; Banks, C. E.; Ward-Jones, S.; Davies, T. J.; Lawrence, N. S.; Jones, T. G. J.; Jiang, L.; Compton, R. G. Boron-doped Diamond Microdisc Arrays: Electrochemical Characterisation and Their Use as a Substrate for The Production of Microelectrode Arrays of Diverse Metals (Ag, Au, Cu) via Electrodeposition. Analyst 2005, 130, 1303-1311.

18. Davies, T.J.; Compton, R. G. The Cyclic and Linear Sweep Voltammetry of Regular and Random Arrays of Microdisc Electrodes: Theory. J. Electroanal. Chem. 2005, 585, 63-82.

19. Cięciwa, A.; Wuthrich, R.; Comninellis, C. Electrochemical Characterization of Mechanically Implanted Boron-doped Diamond Electrodes. Electrochem. Commun. 2006, 8, 375-382.

20. Soh, K.L.; Kang, W.P.; Davidson, J.L.; Basu, S.; Wong, Y.M.; Cliffel, D.E.; Bonds, A.B.; Swain, G.M. Diamond-derived Microelectrodes Array for Electrochemical Analysis. Diam. Relat. Mater. 2004, 13, 2009-2015.

21. Feeney, R.; Kounaves S. P. Microfabricated Ultramicroelectrode Arrays: Developments, Advances, and Applications in Environmental Analysis. Electroanalysis 2000, 12(9), 677-684.

22. Fletcher, S.; Horne, M. D. Random Assemblies of Microelectrodes (RAM electrodes) for Electrochemical Studies. Electrochem. Commun. 1999, 1, 502-512.

23. Lawrence, N. S.; Pagels, M.; Meredith, A.; Jones, T. G. J.; Hall, C.E.; Pickles C. S. J.; Godfried, H. P.; Banks, C. E.; Compton R. G.; Jiang, L. Electroanalytical Applications of Boron-doped Diamond Microelectrode Arrays. Talanta 2006, 69, 829-834.

24. Lee, H. J.; Beriet, C.; Ferrigno, R.; Girault, H., Cyclic Voltammetry at Regular Microdisc Electrode Array, J. Electroanal. Chem. 2001, 502, 138-145.

(C) 2007 by MDPI (http://www.mdpi.org). Reproduction is permitted for noncommercial purposes. 